\title{
Myocardial fibrosis by late gadolinium enhancement cardiovascular magnetic resonance in myotonic muscular dystrophy type 1: highly prevalent but not associated with surface conduction abnormality
}

\author{
Andrea Cardona ${ }^{1,2}$, William D. Arnold ${ }^{3}$, John T. Kissel ${ }^{3}$, Subha V. Raman ${ }^{1}$ and Karolina M. Zareba ${ }^{1 *}$ (D)
}

\begin{abstract}
Background: Conduction disease and arrhythmias represent a major cause of mortality in myotonic muscular dystrophy type 1 (MMD1). Permanent pacemaker (PPM) implantation is the cornerstone of therapy to reduce cardiovascular mortality in MMD1. Cardiovascular magnetic resonance (CMR) studies demonstrate a high prevalence of myocardial fibrosis in MMD1, however the association between CMR myocardial fibrosis with late gadolinium enhancement (CMRLGE) and surface conduction abnormality is not well established in MMD1.

We investigated whether myocardial fibrosis by CMR-LGE is associated with surface conduction abnormalities meeting criteria for PPM implantation according to current guidelines in a cohort of patients with genetically confirmed MMD1.

Methods: Patients with genetically confirmed MMD1 were retrospectively evaluated. 12-lead electrocardiography (ECG) performed within 6 months of CMR was necessary for inclusion. The severity and extent of MMD1 was quantified using a validated Muscular Impairment Rating Scale (MIRS). Based on current guidelines for device-based therapy of cardiac rhythm abnormalities, we defined surface conduction abnormality as the presence of ECG alterations meeting criteria for PPM implant (class I or II indications): PR interval > 200 ms (type I atrioventricular (AV) block) and/or mono or bifascicular block (QRS > $120 \mathrm{~ms}$ ), or evidence of advanced AV block. Balanced steady-state free precession sequences (bSSFP) were used for assessment of left ventricular (LV) volumes and ejection fraction. MOdified Look-Locker Inversion Recovery (MOLLI) acquisition schemes were used to acquire T1 maps. Patients' charts were reviewed up to 12 months post-CMR for occurrence of PPM implantation.

Results: Fifty-two patients (38\% male, $41 \pm 14$ years) were included. Overall, 31 (60\%) patients had a surface conduction abnormality and 22 (42\%) demonstrated midwall myocardial fibrosis by CMR-LGE. After a median of 57 days from CMR exam, 15 patients (29\%) underwent PPM implantation. Subjects with vs. without surface conduction abnormality had significantly longer disease length (15.5 vs. 7.8 years, $p=0.015)$ and higher disease severity on the MIRS scale $(p=0.041)$. High prevalence of myocardial fibrosis by CMR-LGE was detected in subjects with and without surface conduction abnormality with no significant difference between the two cohorts ( $42 \%$ vs. $43 \%, p=0.999)$. By multivariate logistic regression analysis, disease length was the only independent variable associated with surface conduction abnormality (OR 1.071, 95\%Cl 1.003-1.144, $p=0.040)$; while CMR-LGE was not associated with conduction abnormality $(\rho=-0.009$, $p=0.949)$.

(Continued on next page)
\end{abstract}

\footnotetext{
* Correspondence: kzarebamd@gmail.com

${ }^{1}$ Division of Cardiovascular Medicine, The Ohio State University Wexner

Medical Center, 473 W 12th Ave, Suite 200, Columbus OH 43210, USA

Full list of author information is available at the end of the article
}

(c) The Author(s). 2019 Open Access This article is distributed under the terms of the Creative Commons Attribution 4.0 International License (http://creativecommons.org/licenses/by/4.0/), which permits unrestricted use, distribution, and reproduction in any medium, provided you give appropriate credit to the original author(s) and the source, provide a link to the Creative Commons license, and indicate if changes were made. The Creative Commons Public Domain Dedication waiver (http://creativecommons.org/publicdomain/zero/1.0/) applies to the data made available in this article, unless otherwise stated. 
(Continued from previous page)

Conclusions: Myocardial fibrosis by CMR-LGE is highly prevalent in MMD1 but not related to surface conduction abnormality meeting current guideline criteria for PPM implantation .

Keywords: Myotonic muscular dystrophy, Cardiovascular magnetic resonance, Myocardial fibrosis, Electrocardiogram, Pacemaker, Late gadolinium enhancement

\section{Introduction}

Myotonic muscular dystrophy type 1 (MMD1) is the most common muscular dystrophy in adults and is characterized by progressive muscle degeneration leading to disabling weakness and wasting with myotonia, in combination with multisystem involvement [1]. MMD1 is characterized by an autosomal dominant inheritance [2]. CTG repeat expansion in the myotonic dystrophy protein kinase gene (DMPK) is the mutation underlying this condition.

Conduction abnormalities and cardiac arrhythmias represent a major cause of mortality in MMD1; thus the prevention of sudden cardiac death (SCD) is central to patient management [3-6]. Cardiac involvement initially manifests as asymptomatic electrocardiographic (ECG) abnormalities, typically prolongation of the PR and QRS intervals progressing to more advanced conduction disease including sinus node dysfunction and heart block as well as atrial tachycardia, and ventricular tachycardia or fibrillation [3-5]. Progression of conduction system disease to complete atrioventricular (AV) block is the presumed cause of SCD in a high proportion of patients [3-5]. Implantation of a permanent pacemaker (PPM) has been found useful even in asymptomatic patients with an abnormal resting ECG or with HV interval prolongation during electrophysiological study as the disease course can have unpredictable progression to advanced conduction disease $[5,6]$. Therefore, permanent pacing has been recommended by the American College of Cardiology and the American Heart Association when complete AV block or advanced high-degree AV block are detected (class I indication), or prophylactically for patients presenting with first-degree AV or fascicular block on the ECG (class IIb indication) [5].

Myocardial fibrosis has been identified in autopsies from patients with MMD1 together with fatty infiltration and myocyte hypertrophy and degeneration $[7,8]$. Cardiac fibrosis and fatty infiltration most commonly affect the myocardium and the His-Purkinje system, but may also involve the sino-atrial and AV nodes thus providing a substrate for conduction abnormalities and arrhythmias [7, 9-13]. Cardiovascular magnetic resonance (CMR) studies using late gadolinium enhancement imaging (CMR-LGE), where abnormal appearance of the myocardium often represents fibrosis, have revealed a prevalence of myocardial fibrosis by LGE in MMD ranging from 0 to $40 \%$ [9-14]. However, variable associations between conduction abnormalities and myocardial fibrosis by CMR-LGE have been reported [9-13]. To date, there are no studies that have specifically explored the relationship between myocardial fibrosis by CMR-LGE and surface conduction abnormalities meeting criteria for PPM implant in MMD1, a procedure that is advocated for SCD prevention in patients with this disease $[4,5]$.

We sought to evaluate the association between the presence and extent of CMR-LGE myocardial fibrosis and surface conduction abnormalities meeting criteria for PPM implantation according to current guidelines in a cohort of patients with genetically confirmed MMD1.

\section{Methods \\ Population selection}

We retrospectively evaluated consecutive patients from the Muscular Dystrophy Clinic of The Ohio State University Medical Center, with genetically confirmed diagnosis of MMD1 referred for a clinical CMR between April 2012 and March 2017. Subsequent decision-making regarding PPM implantation was done at the clinical discretion of the referring provider, typically based on ECG findings, and did not incorporate CMR data. Standard 12-lead ECG performed within 6 months of CMR exam was necessary for inclusion. Exclusion criteria included standard contraindications to perform a CMR exam: severe claustrophobia, presence of a pacing device, ferromagnetic foreign body, active implant, known allergy to gadolinium-based contrast, or advanced kidney disease (e.g. glomerular filtration rate $<30 \mathrm{~mL} / \mathrm{min} / 1.73 \mathrm{~m} 2$ ). The Ohio State University Institutional Review Board approved this retrospective study and waived informed consent.

\section{Clinical assessment}

All patients underwent a comprehensive clinical evaluation and physical examination during their clinical visit. The severity and extent of MMD was quantified using a validated Muscular Impairment Rating Scale (MIRS) related to the clinical distribution and extent of muscle weakness and myotonia [15]. The New York Heart Association (NYHA) classification system was used to assess exercise capacity. Patient's charts were reviewed up to 12 months after CMR to record date of PPM implant or occurrence of any other major adverse cardiac events defined as heart failure, ventricular arrhythmias, or death. 


\section{Electrophysiologic analysis}

Electrical parameters were assessed from a standard 12-lead surface ECG. Based on current American College of Cardiology Foundation/ American Heart Association/ Heart Rhythm Society (ACCF/AHA/HRS) guidelines for device-based therapy of cardiac rhythm abnormalities [5], we defined surface conduction abnormality as the presence of ECG alterations meeting criteria for PPM implant (class I or II indications) in subjects with MMD. Accordingly, surface conduction abnormality was defined as evidence of PR interval $>200$ ms (type I AV block) and/or mono or bifascicular block (QRS $>120 \mathrm{~ms}$ ), or evidence of an advanced AV block [5]. Left ventricular (LV) hypertrophy (LVH) was assessed by Cornell criteria [16]. According to current consensus documents for Standardization and Interpretation of the Electrocardiogram from the ACCF/AHA/HRS, standard criteria were adopted to define the presence of left anterior fascicular block (LAFB), left posterior fascicular block (LPFB), left bundle branch block (LBBB), right bundle branch block (RBBB), and non-specific intraventricular conduction delay (IVCD) [17]. The QT interval was obtained via automated ECG analysis in leads II, V2, and V5. The QTc interval was calculated using the Bazett formula. Electrophysiological (EP) study data and pacing device information were retrieved from patients' charts. Referrals for intracardiac EP studies were performed in the presence of symptomatic or asymptomatic AV conduction abnormalities (first-degree or higher AV block) with or without QRS interval $\geq 120 \mathrm{~ms}$, and/or presence of palpitations, syncope, near syncope or documented arrhythmias.

\section{Cardiovascular magnetic resonance protocol}

All images were acquired on a single 1.5T CMR scanner with a 12-element phased array chest coil (MAGNETOM Avanto, Siemens Healthineers, Erlangen, Germany). Balanced steady-state free precession sequences (bSSFP) were used for assessment of LV volumes, ejection fraction (LVEF) and LV mass. LGE imaging was performed using a gradient-echo inversion recovery sequence with magnitude and phase sensitive inversion recovery reconstructions $10 \mathrm{~min}$ after 0.2 $\mathrm{mmol} / \mathrm{kg}$ of gadolinium based contrast agent [18]. LV volumes and LVEF were measured from contiguous LV short-axis cine images using semi-automated software for endocardial segmentation using endocardial and epicardial contours at end-systole and end-diastole with Simpson's rule. LV mass was calculated from the total end-diastolic myocardial volume multiplied by the specific gravity of the myocardium $(1.05 \mathrm{~g} / \mathrm{ml})$ [19]. Presence of LGE was assessed by 2 expert operators blinded to clinical data (AC, KZ), with a third providing adjudication if necessary (SVR). The LGE (grams) mass was quantified by a blinded operator (AC) using a dedicated software implementing the full-width at half-maximum technique (CMR42, Circle Cardiovascular Imaging Inc. Calgary, Alberta, Canada) and indexed as a percentage of LV mass [20]. MOdified Look-Locker Inversion Recovery (MOLLI) acquisition schemes were used to acquire T1 maps produced using vendor software before and $15 \mathrm{~min}$ after administration of contrast. The MOLLI 3(3)3(3)5 sequence was used in the first 23 patients and subsequently the MOLLI 5(3)3 sequence was used in 29 patients. T1 values and extracellular volume fraction (ECV) were measured and calculated utilizing interventricular septal values from the mid short axis view. The region of interest was placed in the mid myocardium with manual tracing to avoid partial volume effects and regions of LGE if present [21, 22]. Myocardial ECV was calculated as previously described [23]. Reference values for normal pre- and post-contrast myocardial T1 values and ECV for the MOLLI 3(3)3(3)5 sequence are based on data of 18 healthy subjects at our institution ( $45 \pm 18$ years, $39 \%$ female), and are as follows: native T1 $940 \pm 28 \mathrm{~ms}$, post-contrast T1 $403 \pm 42 \mathrm{~ms}$, and ECV $24.3 \pm 2.3 \%$. Reference values for the myocardium utilizing the MOLLI 5(3)3 sequence based on 44 healthy subjects at our institution ( $36 \pm 15$ years, $59 \%$ female) are as follows: native T1 $999 \pm 31 \mathrm{~ms}$, post-contrast T1 $453 \pm$ $30 \mathrm{~ms}$, and ECV $23.8 \pm 2.6 \%$. In line with previous literature, the abnormal value for ECV was $29 \%$, corresponding to $2 \mathrm{SD}$ above the reference value [24].

\section{Statistical analysis}

Data are presented as mean \pm standard deviation (SD) or as median and interquartile range (IQR) for continuous variables and as proportions for categorical variables. The mean values of continuous, normally distributed variables were compared with the 2-sample t-test. Comparison of median values or proportions was done with the 2-sample Wilcoxon rank-sum test, and Fisher exact test, respectively. Univariate logistic regression analysis was used to find individual variables associated with surface conduction abnormality. After testing for collinearity, multivariate stepwise-backward logistic regression analysis was used to find independent variables associated with surface conduction abnormality. The association between CMR-LGE, surface conduction abnormality and PPM implant was also assessed with Spearman correlation analysis. An additional analysis was performed to compare clinical and ECG characteristics according to the presence vs. absence of LGE. Statistical significance was set at two tailed $p<0.05$. SPSS Statistic 21.0 (Statistical Package for the Social Sciences (SSPS) International Business Machines, Inc., Armonk, New York, USA) was used for all statistical analyses. 


\section{Results}

\section{Patients characteristics}

Fifty-two patients with genetically confirmed MMD1 (median CTG repeats $=500, \mathrm{IQR}=200-1163$ ), 38\% male, $41 \pm 14$ years, met criteria for inclusion in the study. Overall, thirty-one patients (60\%) demonstrated surface conduction abnormality: 20 (38\%) with a prolonged PR interval (mean $232 \pm 51 \mathrm{~ms}) ; 5(9.6 \%)$ with a prolonged PR interval associated with RBBB; 2 (3.8\%) patients had a prolonged PR interval associated with LBBB; 2 (3.8\%) had LBBB alone, one patient had RBBB alone, and one had Mobitz AV block type I. Baseline demographic, clinical and ECG characteristics according to presence or absence of surface conduction abnormality are presented in Table 1.

Subjects with conduction abnormality tended to be older, had a longer disease length (15.5 vs. 7.8 years, $p=$ $0.015)$ and demonstrated higher MIRS scores $(p=0.041)$ compared to subjects without conduction abnormalities (Table 1). As expected they also demonstrated a longer PR and QRS intervals as compared to subjects without conduction abnormality. Alternatively, no differences were found between the two groups in the prevalence of major cardiovascular risk factors or medications, CTG repeats, or functional status (Table 1 ).

After a median time of 57 days from the CMR exam, 15 patients (29\%) received a PPM: 7 subjects were implanted due to coexistence of first degree AV and fascicular block, 4 for presence of bifascicular block associated with either a first degree AV block or a prolonged HV interval, 2 for a prolonged HV interval, one for presence of second degree AV block (Mobitz type I), and one for presence of I degree AV block (Table 2). No cardiac death or major cardiovascular events occurred during the 12 months of observation.

\section{CMR findings}

Overall, twenty-two subjects demonstrated midwall myocardial fibrosis by CMR-LGE, corresponding to a prevalence of $42 \%$. The LVEF, and LV volumes were within normal range for the entire population (LVEF 60 $\pm 6 \%$, LV end-diastolic volume index $65 \pm 15 \mathrm{ml} / \mathrm{m}^{2}, \mathrm{LV}$ end-systolic volume index $26 \pm 8 \mathrm{ml} / \mathrm{m}^{2}$ ) (Table 3 ).

The median time between the CMR exam and the ECG was 21 days (IQR 7-54). Subjects with conduction abnormality demonstrated similar LVEF, LV volumes, LV mass, and left atrial (LA) volumes compared to those without conduction abnormality (Table 3). Importantly, high prevalence of myocardial fibrosis by CMR-LGE was detected in subjects with and without surface conduction abnormality but no significant difference was noted between the two groups ( $42 \%$ vs. $43 \%, p=0.999)$. Further, LGE mass and LGE\% were similar between the two groups. ECV tended to be higher in the conduction abnormality group, albeit values were within the normal range in both groups ( $26 \pm 3$ vs. $24 \pm 3, p=0.050$ ). Data on native myocardial and post-contrast T1 values according to different MOLLI sequences and presence vs. absence of conduction abnormality are presented in Additional file 1: Table S1.

Of 22 LGE-positive subjects, all demonstrated midwall fibrosis; in 17 subjects (77\%) the interventricular septum was involved, the inferior and inferolateral segments were involved in four subjects (18\%), and the anterior wall was involved in one subject (5\%). An analysis of clinical and ECG data according to the presence vs. absence of LGE was also conducted (Additional file 1: Tables S2 and S3). There were no significant differences in ECG characteristics in LGE positive vs.LGE negative patients. Fig. 1 demonstrates representative ECG and CMR findings in 2 patients. Additionally, we have provided a Additional file 1: Figure. S1 which includes representative ECG and T1 maps in 2 patients.

\section{Variables associated with surface conduction abnormality} Disease length and Cornell voltage were associated with surface ECG conduction abnormality on univariate analysis. Disease length was the only independent variable associated with conduction abnormality in the multivariate model (OR 1.071, 95\%CI 1.003-1.144, $p=0.040$; Table 4). Importantly, the presence of myocardial fibrosis by CMR-LGE, LGE mass, or LGE\% was not associated with surface conduction abnormality (full regression analysis is shown in Additional file 1: Table S4). Additionally, myocardial fibrosis by CMR-LGE was not associated with surface conduction abnormality by Spearman correlation analysis $(\rho=-0.009, p=0.949)$ nor was LGE\% $(\rho=0.004$, $p=0.976)$ or LGE mass $(\rho=-0.022, p=0.878)$. Similar findings were also true for the association between CMR-LGE and PPM implant $(\rho=-0.03, p=0.834)$.

\section{Discussion}

Our study demonstrates that myocardial fibrosis assessed by CMR-LGE is highly prevalent in patients with MMD1 but is not associated with surface conduction abnormality meeting criteria for PPM implant per current guidelines. An important aspect of our study is the high prevalence of myocardial fibrosis by CMR-LGE in subjects without surface conduction abnormality. This relevant finding deserves further attention to better understand the role of CMR in risk stratification of patients with myotonic dystrophy in longer-term follow-up.

In MMD1, conduction system abnormalities can evolve into complete AV block and can be associated with potentially fatal asystole or ventricular arrhythmias that result in SCD [3, 4, 25-27]. However, the cause of a dysfunctional conduction system in MMD is yet to be determined. Myocardial fibrosis can affect the structural integrity and electrical conductive properties of cardiac muscle leading to 
Table 1 Demographic, clinical and electrocardiographic findings in the study cohort

\begin{tabular}{|c|c|c|c|c|}
\hline & Whole Cohort $(N=52)$ & $\begin{array}{l}\text { Conduction Abnormality } \\
\text { Positive }(N=31)\end{array}$ & $\begin{array}{l}\text { Conduction Abnormality } \\
\text { Negative }(N=21)\end{array}$ & $P$ valu \\
\hline \multicolumn{5}{|l|}{ Demographic data } \\
\hline Age, years & $41.2 \pm 13.9$ & $43.7 \pm 11.9$ & $37.4 \pm 15.9$ & 0.129 \\
\hline Male gender, N (\%) & $20(38)$ & $11(35)$ & $9(43)$ & 0.772 \\
\hline $\mathrm{BMl}, \mathrm{kg} / \mathrm{m}^{2}$ & $25.5 \pm 5.8$ & $25.8 \pm 6.2$ & $24.9 \pm 5.1$ & 0.618 \\
\hline $\mathrm{DM}, \mathrm{N}(\%)$ & $5(10)$ & $4(13)$ & $1(5)$ & 0.637 \\
\hline HTN, N (\%) & $3(6)$ & $1(3)$ & $2(9)$ & 0.558 \\
\hline Smoking, N (\%) & $2(4)$ & $2(6)$ & $0(0)$ & 0.494 \\
\hline HLP, N (\%) & $5(10)$ & $4(13)$ & $1(5)$ & 0.637 \\
\hline History of CAD, N (\%) & $1(2)$ & $1(3)$ & $0(0)$ & 0.999 \\
\hline BB, N (\%) & $4(8)$ & $3(10)$ & $1(5)$ & 0.639 \\
\hline ACE-i, N (\%) & $3(6)$ & $2(6)$ & $1(5)$ & 0.999 \\
\hline ARB, N (\%) & $1(2)$ & $1(3)$ & $0(0)$ & 0.999 \\
\hline MCRA, N (\%) & $4(8)$ & $4(13)$ & $0(0)$ & 0.138 \\
\hline Statin, N (\%) & $6(11)$ & $4(13)$ & $2(9)$ & 0.999 \\
\hline
\end{tabular}

Myotonic Muscular Dystrophy Characteristics

$\begin{array}{lll}\text { Age of onset, years } & 28.8 \pm 15.5 & 28.2 \pm 17.1 \\ \text { Disease length, years } & 12.4 \pm 12.7 & 15.5 \pm 14.8 \\ \text { CTG Repeats } & 500(200-1163) & 280(142-772 \\ \text { MIRS scale } & 3(3-4) & 3(3-4)\end{array}$

$\begin{array}{ll}29.6 \pm 13.1 & 0.749 \\ 7.8 \pm 6.9 & 0.015^{*} \\ 575(450-1450) & 0.137 \\ 3(2-3) & 0.041^{*}\end{array}$

Clinical data

$\begin{array}{lllll}\text { SBP, mmHg } & 126 \pm 18 & 125 \pm 18 & 126 \pm 19 & 0.798 \\ \text { DBP, mmHg } & 74 \pm 10 & 74 \pm 11 & 75 \pm 8 & 0.733 \\ \text { HR, bpm } & 74 \pm 14 & 74 \pm 16 & 75 \pm 12 & 0.793 \\ \text { NYHA class } & 2(1-2) & 2(1-2) & 1(1-2) & 0.900 \\ \text { Hematocrit, \% } & 42.1 \pm 3.8 & 42.3 \pm 3.4 & 41.7 \pm 4.4 & 0.592\end{array}$

Electrocardiographic Data

\begin{tabular}{lllll} 
PR, ms & $199 \pm 47$ & $223 \pm 48$ & $165 \pm 16$ & 0.001 \\
QRS, ms & $103 \pm 20$ & $111 \pm 22$ & $94 \pm 11$ & 0.001 \\
QT, ms & $404 \pm 42$ & $412 \pm 47$ & $392 \pm 31$ & 0.070 \\
QTc, ms & $428 \pm 32$ & $431 \pm 38$ & $423 \pm 23$ & 0.359 \\
Frontal QRS-T angle & $41 \pm 41$ & $45 \pm 44$ & $36 \pm 36$ & 0.447 \\
LVH-Cornell, N (\%) & $13(25)$ & $11(35)$ & $2(9)$ & 0.050 \\
\hline
\end{tabular}

Data are presented as mean $\pm \mathrm{SD}, \mathrm{N}(\%)$, or median (interquartile range). ${ }^{*} p<0.05$ considered significant. $B M I$ body mass index, $D M$ diabetes mellitus, $H L P$ hyperlipidemia, $C A D$ coronary artery disease, $B B$ beta-blockers, $A C E-i$ Angiotensin-converting enzyme inhibitors, $A R B$ Angiotensin receptor blockers, $M C R A$ aldosterone antagonists, MIRS Muscular Impairment Rating Scale, SBP systolic blood pressure, DBP diastolic blood pressure, HR heart rate, NYHA New York Heart Association class, LVH left ventricular hypertrophy

heart failure and arrhythmias [28, 29]. However, the association between myocardial fibrosis and surface conduction abnormalities in MMD1 has not been well defined and contrasting evidence exists in this regard $[10,11]$. In a previous CMR study including 80 patients with MMD1 myocardial fibrosis was associated with concomitant abnormal ECG; however, the prevalence of fibrosis was rather low (13\%) [10]. A subsequent study involving 30 subjects 


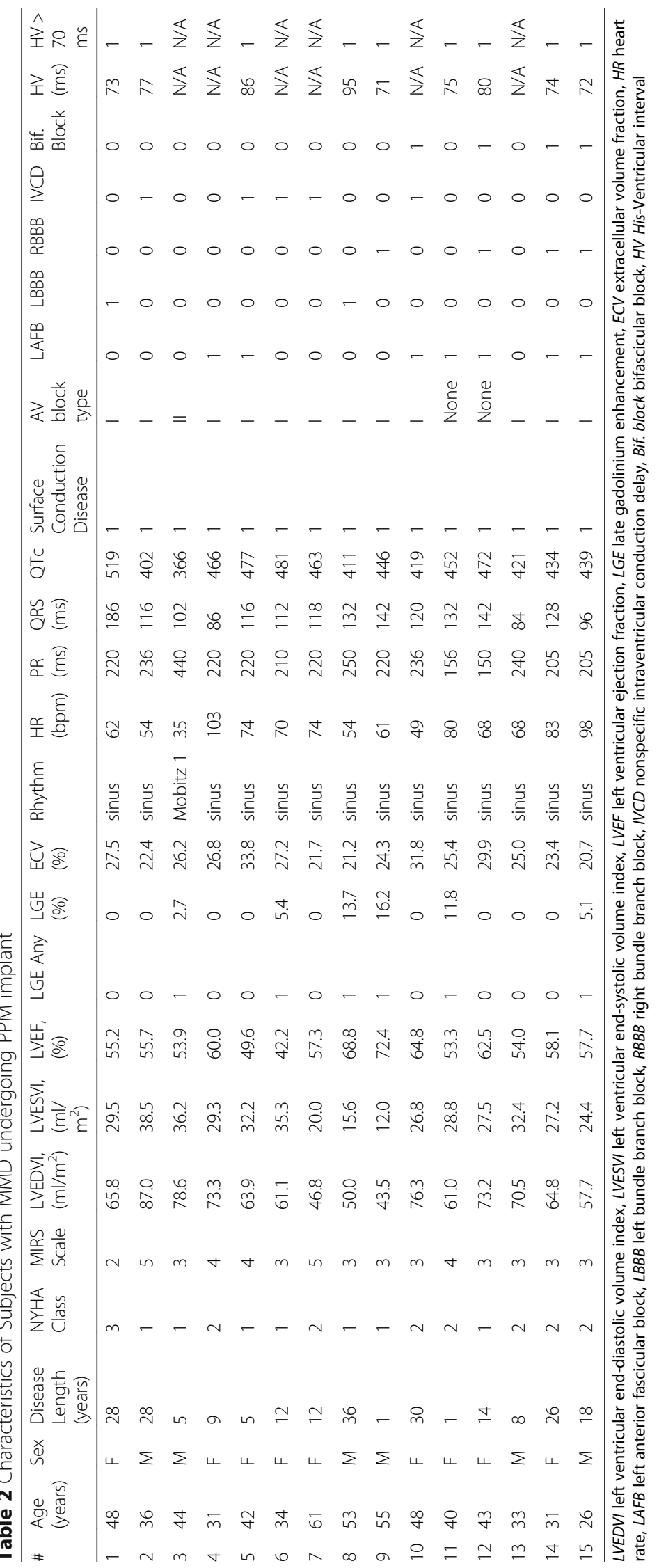


Table 3 CMR Findings

\begin{tabular}{|c|c|c|c|c|}
\hline CMR data & Whole Cohort $(N=52)$ & $\begin{array}{l}\text { Conduction Abnormality } \\
\text { Positive }(N=31)\end{array}$ & $\begin{array}{l}\text { Conduction Abnormality } \\
\text { Negative }(N=21)\end{array}$ & $P$ value \\
\hline LVEF (\%) & $60 \pm 6$ & $59 \pm 6$ & $60 \pm 6$ & 0.687 \\
\hline LV EDVI, $\mathrm{ml} / \mathrm{m}^{2}$ & $65 \pm 15$ & $65 \pm 14$ & $66 \pm 15$ & 0.728 \\
\hline LV ESVI, $\mathrm{ml} / \mathrm{m}^{2}$ & $26 \pm 8$ & $26 \pm 8$ & $26 \pm 7$ & 0.940 \\
\hline LV mass index, $\mathrm{g} / \mathrm{m}^{2}$ & $44 \pm 11$ & $45 \pm 14$ & $43 \pm 6$ & 0.563 \\
\hline $\mathrm{LAVl}, \mathrm{ml} / \mathrm{m}^{2}$ & $30 \pm 11$ & $31 \pm 10$ & $29 \pm 12$ & 0.634 \\
\hline LGE, N (\%) & $22(42 \%)$ & $13(42)$ & $9(43)$ & 0.999 \\
\hline LGE mass, $g$ & $2.4 \pm 3.6$ & $2.4 \pm 3.8$ & $2.3 \pm 3.4$ & 0.876 \\
\hline LGE, \% & $3.1 \pm 4.6$ & $3.0 \pm 4.6$ & $3.1 \pm 4.7$ & 0.975 \\
\hline $\mathrm{ECV}, \%$ & $25 \pm 3$ & $26 \pm 3$ & $24 \pm 3$ & 0.050 \\
\hline
\end{tabular}

Data are presented as mean \pm SD or $\mathrm{N}(\%)$. LVEF left ventricular ejection fraction, EDVI end-diastolic volume index, ESVI end-systolic volume index, LAVI left atrial volume index, $L G E$ late gadolinium enhancement, ECV extracellular volume

with MMD1, showed a higher prevalence of myocardial fibrosis (40\%) and no association with abnormal ECG findings [9]. Our findings confirm a high prevalence of myocardial fibrosis (42\%) with no association with surface conduction abnormalities in a large MMD1 population. Furthermore, we found no association between myocardial fibrosis by CMR-LGE and PPM implant which is a major clinical need for SCD prevention in MMD.

In our study the distribution of midwall myocardial fibrosis by LGE-CMR was predominantly in the septum (77\%) followed by inferior and inferolateral regions (18\%) - these findings are consistent with prior literature. In the study by Petri et al. there were 12 patients with LGE positivity, 50\% exhibited fibrosis in the septum (anteroseptum and hinge points) and 33\% in the inferior and inferolateral regions [9]. Similarly, Hermans and colleagues noted that 8 of the 10 patients with LGE positivity exhibited fibrosis in the septum and inferolateral wall [10]. In light of recent literature in the non-ischemic population demonstrating even a small extent of septal LGE being associated with increased risk of adverse cardiovascular events [30], the LGE extent and distribution in MMD1 patients raise concern for the arrhythmogenic potential of myocardial fibrosis in this population and further solidify the role of CMR in MMD1.

Our study suggests that myocardial fibrosis and conduction system abnormality may be two distinct phenomena occurring simultaneously in the myocardium of subjects affected by MMD1 and possibly carrying an interrelated but independent risk for adverse cardiovascular events. Recent studies seem to support this hypothesis, suggesting a possible relation between molecular mechanisms and surface conduction abnormalities [31, 32]. An analysis performed in subjects with MMD1 demonstrated a significant reduction in the expression of a microRNA precursor (miR-1) leading to deregulation of two important miR-1 targets: Connexion 43 and cardiac L-type calcium channel which encode gap-junction and the main calcium channels in the heart, respectively. Their misregulation may contribute to the conduction abnormalities observed in this population.

An additional aspect of our study is data on both replacement and interstitial fibrosis. In this cohort of MMD1 patients, we identified and quantified replacement fibrosis by LGE, and quantified interstitial fibrosis utilizing T1 mapping to obtain ECV values. Although there was a trend towards higher ECV values in patients with surface conduction abnormality, ECV was not statistically significantly different between the two groups. Further studies are needed to elucidate the nature of interstitial fibrosis in MMD1 [22].

Another important result of our study is the demonstration of high prevalence of myocardial fibrosis by CMR-LGE in subjects without surface conduction abnormality (43\%). This finding has two relevant implications: i) myocardial fibrosis by CMR-LGE and surface conduction abnormality can occur independently in MMD and ii) additional studies are needed to know if detection of fibrosis afforded by CMR-LGE helps in predicting tachyarrhythmia risk in MMD that may not be captured by conduction abnormalities. The presence of myocardial fibrosis by CMR-LGE plays a key role in multiple cardiomyopathies as an important prognosticator for worse cardiovascular outcomes, particularly ventricular arrhythmias and sudden cardiac death [33-37]. In patients with MMD, myocardial fibrosis has been observed both in CMR and autopsy studies, giving evidence of a possible link between myocardial fibrosis and SCD independently of surface conduction abnormality [9-11]. Therefore, the high prevalence of myocardial fibrosis by CMR-LGE in the absence of surface conduction abnormality supports CMR's complementary role for risk stratification in these patients. Furthermore, the presence of myocardial fibrosis by CMR could guide early initiation of cardioprotective therapies aimed to 

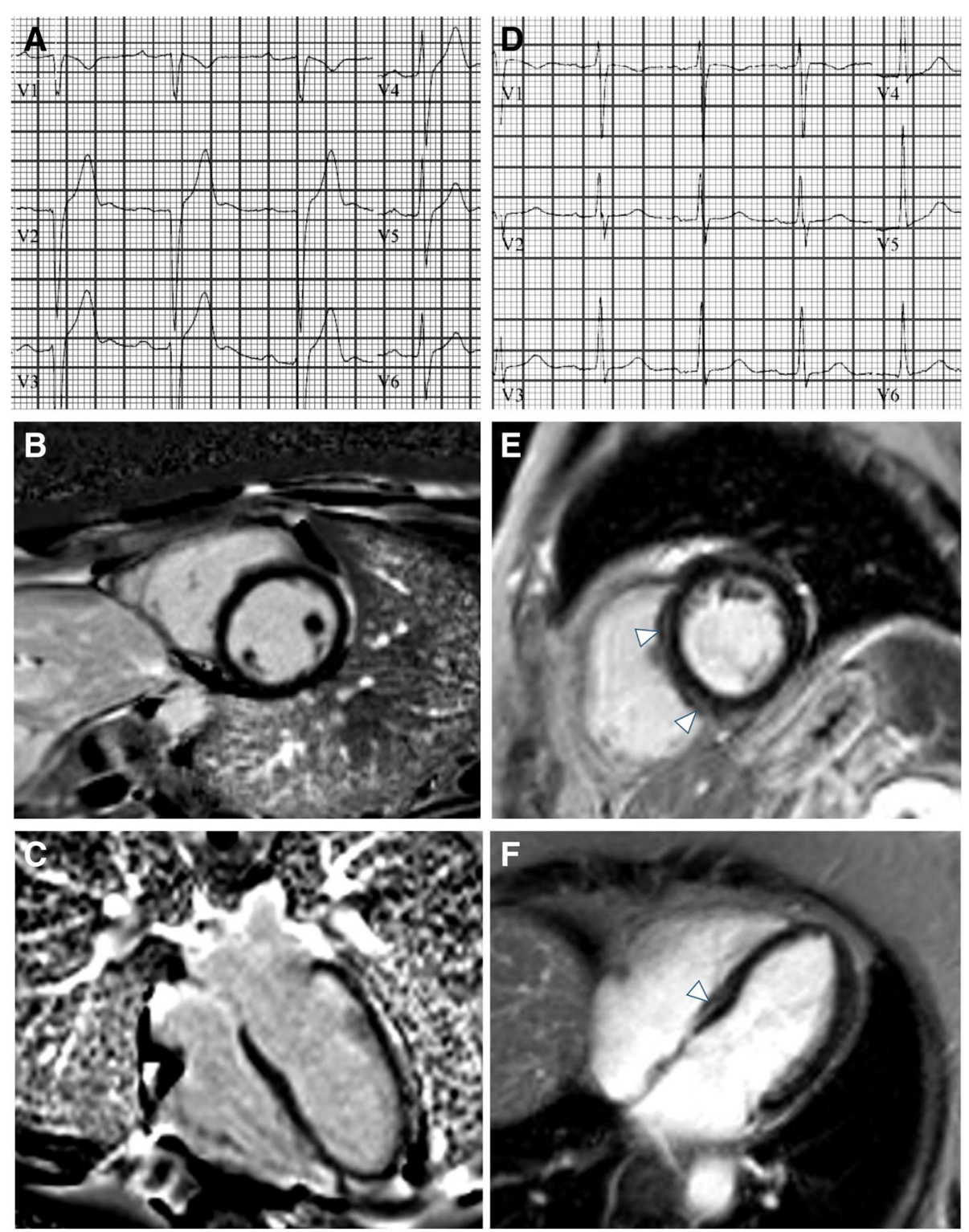

Fig. 1 Representative ECG and CMR findings in the study cohort. Panels A shows an abnormal ECG with prolonged PR interval and borderline QRS interval of a subject with no evidence of myocardial fibrosis by CMR-LGE (B, C). Panels D shows a normal ECG of a subjects with evidence of midwall fibrosis mostly evident in the interventricular septum (arrows in $\mathrm{E}$, and $\mathrm{F}$ )

reduce the fibrotic burden and subsequent LV dysfunction as has been validated in other forms of neuromuscular disease [38].

Finally, similar to MMD1, MMD type 2 also manifests with cardiac involvement [39, 40]. Conduction abnormalities present in a similar pattern to MMD1, although they are less prevalent and appear to be more variable between affected individuals. CMR also demonstrates abnormalities in LGE and T1 signal in MMD2 individuals $[14,40]$. Further larger studies are needed to establish the relationship between conduction and CMR abnormalities in MMD2.

\section{Limitations}

This is a single center, retrospective study and the results should be confirmed in a large, multicenter, prospective study powered for both bradyarrhythmias as well as tachyarrhythmic SCD events.

It could be speculated that lack of correlation between myocardial fibrosis by CMR-LGE with surface conduction abnormality could be related to relatively early course of disease from the point of view of myocardial structural abnormality; however our findings rather support the opposite conclusion for the following reasons: i) there is a high prevalence of 
Table 4 Variables Associated with Surface Conduction Abnormality

\begin{tabular}{|c|c|c|c|}
\hline Variables & $\operatorname{Exp}(\mathrm{B})$ or OR & $95 \% \mathrm{Cl}$ & $P$ value \\
\hline \multicolumn{4}{|l|}{ Univariate Analysis } \\
\hline Disease length, years & 1.071 & $1.003-1.144$ & $0.040^{*}$ \\
\hline Cornell voltage, mm & 1.090 & $1.007-1.179$ & $0.033^{*}$ \\
\hline Presence of CMR-LGE & 0.963 & $0.314-2.953$ & 0.947 \\
\hline \multicolumn{4}{|l|}{ Multivariate Analysis ${ }^{a}$} \\
\hline Disease length, years & 1.071 & $1.003-1.144$ & $0.040^{*}$ \\
\hline
\end{tabular}

myocardial fibrosis which demonstrates that myocardial structural abnormality was frequent in this population ii) LV mass index was in the low normal range, which in line with previous studies, confirms evident muscle wasting typical for MMD1 iii) finally the length of MMD1 (median >12 years in the whole population) excludes the possibility of an early stage of the disease where myocardial structural abnormality could have been absent or not detectable with CMR. Although we did not see trends towards significance with respect to myocardial fibrosis by CMR-LGE between our two cohorts, and our study is one of the largest CMR based studies in MMD1, our acceptance of the null hypothesis may relate to our sample size.

As the intent of our manuscript was to determine the association between myocardial fibrosis by CMR-LGE and surface conduction abnormality, we retrospectively included all consecutive patients with contrasted CMR exams and ECGs performed within 6 months. Our study spanned nearly 5 years during which two different MOLLI sequences were used at our institution, MOLLI 3(3)3(3) 5 initially and then MOLLI 5(3)3. This is a limitation of our study.

ECV values were calculated from ROIs placed in the interventricular septum from the mid short axis view, hence they are not representative of the whole myocardium. However, according to recent literature and consensus documents, septal segments have proven to be the most reproducible as they are less frequently affected by off-resonance artifacts [21, 22]. Additionally, basal short axis T1 maps were not obtained at the time of the clinical scan, thus our data is only representative of the mid-short axis slice.

\section{Conclusion}

Myocardial fibrosis by CMR-LGE in patients with MMD1 is highly prevalent but not related to surface conduction abnormality meeting criteria for PPM implantation according to current guidelines.

Our findings support the concept that MMD1 is characterized by a complex phenotype where conduction system dysfunction and myocardial fibrosis assessed by CMR-LGE are independent phenomena possibly with additive but independent prognostic significance.

High prevalence of myocardial fibrosis by CMR-LGE in the absence of surface conduction abnormalities warrants longer term follow-up to understand how CMR informs risk stratification in this disease.

\section{Additional file}

Additional file 1: Table S1. T1 mapping in patients with and without conduction abnormality. Table S2. Patient characteristics according to CMRLGE findings. Table S3. Electrocardiographic Characteristics According to CMR-LGE findings. Table S4. Full Univariate Logistic Regression Analysis of Variables Associated with Surface Conduction Abnormality. Fig. S1. Representative ECG and CMR findings in the study cohort including T1 mapping. Panels A shows an patient with abnormal ECG with prolonged PR interval and right bundle branch block, his corresponding native T1 map in panel B (utilizing MOLLI 3(3)3(3)5) and post-contrast T1 map in panel $C$ yielding an ECV of 24\%. Panel D demonstrates septal and inferolateral midwall fibrosis in the same patient. Panel E demonstrates a patient without conduction abnormality; his pre- and post-contrast T1 maps in panels $\mathrm{F}$ and

G (utilizing MOLLI 5(3)3) yield an ECV of $23 \%$ and panel $\mathrm{H}$ demonstrates fibrosis in the inferior RV insertion site.

\section{Abbreviation}

AV: Atrioventricular; bSSFP: Balanced steady-state free precession sequences; CMR: Cardiovascular magnetic resonance; DMPK: Dystrophy protein kinase gene; ECG: Electrocardiogram; ECV: Extracellular volume fraction;

EP: Electrophysiological; IVCD: non-specific intraventricular conduction delay; LA: Left atrium/left atrial; LAFB: Left anterior fascicular block; LBBB: Left bundle branch block; LGE: Late gadolinium enhancement; LPFB: Left posterior fascicular block; LV: Left ventricle/left ventricular; LVEF: Left ventricular ejection fraction; LVH: Left ventricular hypertrophy; MIRS: Muscular Impairment Rating Scale; MMD1: Myotonic Muscular Dystrophy type 1; MOLLI: MOdified Look-Locker Inversion Recovery; NYHA: New York Heart Association; PPM: Permanent pacemaker; RBBB: Right bundle branch block; SCD: Sudden cardiac death

\section{Acknowledgements}

Not applicable.

\section{Funding}

Research reported in this publication was supported by the National Institute of Health under award number 1L30HL134084-01, and The Ohio State University Davis Heart and Lung Research Institute Trifit Award. The content is solely the responsibility of the authors and does not necessarily represent the official views of the National Institutes of Health or The Ohio State University.

\section{Availability of data and materials}

The datasets used and analyzed during the current study is available from the corresponding author on reasonable request.

\section{Authors' contributions}

AC: conception and design of study, drafted the manuscript, collection and analysis of the data. WDA: conception and design of study, collection and analysis of the data, critical revision of the manuscript. JTK: conception and design of study, critical revision of the manuscript. SVR: conception and design of study, critical revision of the manuscript, analysis of the data. KZ: conception and design of study, collection and analysis of the data, critical revisions of the manuscript. All authors read and approved the final manuscript. 


\section{Authors' information}

No third parties were involved in the authorship of review of the manuscript.

\section{Consent for publication}

Not applicable. All data presented in this article is non-identifiable.

\section{Competing interests}

The authors have declared that they have no competing interests.

\section{Publisher's Note}

Springer Nature remains neutral with regard to jurisdictional claims in published maps and institutional affiliations.

\section{Author details}

'Division of Cardiovascular Medicine, The Ohio State University Wexner Medical Center, 473 W 12th Ave, Suite 200, Columbus OH 43210, USA. ${ }^{2}$ Division of Cardiology, University of Perugia, Rome, Italy. ${ }^{3}$ Division of Neuromuscular Disorders, The Ohio State University Wexner Medical Center, Columbus, OH, USA.

Received: 15 June 2018 Accepted: 26 March 2019

Published online: 02 May 2019

\section{References}

1. Udd B, Krahe R. The myotonic dystrophies: molecular, clinical, and therapeutic challenges. Lancet Neurol. 2012;11:891-905.

2. Brook JD, McCurrach ME, Harley HG, et al. Molecular basis of myotonic dystrophy: expansion of a trinucleotide (CTG) repeat at the $3^{\prime}$ end of a transcript encoding a protein kinase family member. Cell. 1992;68:799-808.

3. Groh WJ, Groh MR, Saha C, et al. Electrocardiographic abnormalities and sudden death in myotonic dystrophy type 1. N Engl J Med. 2008:358:2688-97.

4. Wahbi K, Meune C, Porcher R, et al. Electrophysiological study with prophylactic pacing and survival in adults with myotonic dystrophy and conduction system disease. JAMA. 2012;307:1292-301.

5. Epstein AE, DiMarco JP, Ellenbogen KA, et al. 2012 ACCF/AHA/HRS focused update incorporated into the ACCF/AHA/HRS 2008 guidelines for devicebased therapy of cardiac rhythm abnormalities: a report of the American College of Cardiology Foundation/American Heart Association Task Force on Practice Guidelines and the Heart Rhythm Society. J Am Coll Cardiol. 2013;61:e6-75.

6. European Heart Rhythm A, Heart rhythm S, Zipes DP, et al. ACC/AHA/ESC 2006 guidelines for management of patients with ventricular arrhythmias and the prevention of sudden cardiac death: a report of the American College of Cardiology/American Heart Association task force and the European Society of Cardiology Committee for practice guidelines (writing committee to develop guidelines for Management of Patients with Ventricular Arrhythmias and the prevention of sudden cardiac death). J Am Coll Cardiol. 2006;48:e247-346.

7. Finsterer J, Stollberger $\mathrm{C}$, Keller H. Arrhythmia-related workup in hereditary myopathies. J Electrocardiol. 2012;45:376-84.

8. Lau JK, Sy RW, Corbett A, Kritharides L. Myotonic dystrophy and the heart: a systematic review of evaluation and management. Int J Cardiol. 2015;184:600-8.

9. Petri H, Ahtarovski KA, Vejlstrup N, et al. Myocardial fibrosis in patients with myotonic dystrophy type 1: a cardiovascular magnetic resonance study. J Cardiovasc Magn Reson. 2014;16:59.

10. Hermans MC, Faber CG, Bekkers SC, et al. Structural and functional cardiac changes in myotonic dystrophy type 1: a cardiovascular magnetic resonance study. J Cardiovasc Magn Reson. 2012;14:48.

11. Choudhary P, Nandakumar R, Greig H, et al. Structural and electrical cardiac abnormalities are prevalent in asymptomatic adults with myotonic dystrophy. Heart. 2016;102:1472-8.

12. Verhaert D, Richards K, Rafael-Fortney JA, Raman SV. Cardiac involvement in patients with muscular dystrophies: magnetic resonance imaging phenotype and genotypic considerations. Circ Cardiovasc Imaging. 2011;4:67-76.

13. Tanawuttiwat T, Wagner KR, Tomaselli G, Nazarian S. Left ventricular dysfunction and conduction disturbances in patients with myotonic muscular dystrophy type I and II. JAMA Cardiol. 2017;2:225-8.

14. Turkbey EB, Gai N, Lima JA, et al. Assessment of cardiac involvement in myotonic muscular dystrophy by $\mathrm{T} 1$ mapping on magnetic resonance imaging. Heart Rhythm. 2012;9:1691-7.
15. Mathieu J, Boivin H, Meunier D, Gaudreault M, Begin P. Assessment of a disease-specific muscular impairment rating scale in myotonic dystrophy. Neurology. 2001;56:336-40

16. Casale PN, Devereux RB, Kligfield P, et al. Electrocardiographic detection of left ventricular hypertrophy: development and prospective validation of improved criteria. J Am Coll Cardiol. 1985;6:572-80.

17. Surawicz B, Childers R, Deal BJ, et al. AHA/ACCF/HRS recommendations for the standardization and interpretation of the electrocardiogram: part III: intraventricular conduction disturbances: a scientific statement from the American Heart Association Electrocardiography and Arrhythmias Committee, Council on Clinical Cardiology; the American College of Cardiology Foundation; and the Heart Rhythm Society Endorsed by the International Society for Computerized Electrocardiology. J Am Coll Cardiol. 2009:53:976-81,

18. Kellman P, Arai AE, McVeigh ER, Aletras AH. Phase-sensitive inversion recovery for detecting myocardial infarction using gadolinium-delayed hyperenhancement. Magn Reson Med. 2002;47:372-83.

19. Reiter G, Reiter U, Rienmuller R, Gagarina N, Ryabikin A. On the value of geometry-based models for left ventricular volumetry in magnetic resonance imaging and electron beam tomography: a bland-Altman analysis. Eur J Radiol. 2004;52:110-8.

20. Flett AS, Hasleton J, Cook C, et al. Evaluation of techniques for the quantification of myocardial scar of differing etiology using cardiac magnetic resonance. JACC Cardiovasc Imaging. 2011;4:150-6.

21. Puntmann VO, Peker E, Chandrashekhar Y, Nagel E. T1 mapping in characterizing myocardial disease: a comprehensive review. Circ Res. 2016; 119:277-99.

22. Moon JC, Messroghli DR, Kellman P, et al. Myocardial T1 mapping and extracellular volume quantification: a Society for Cardiovascular Magnetic Resonance (SCMR) and CMR working Group of the European Society of cardiology consensus statement. J Cardiovasc Magn Reson. 2013;15:92.

23. Messroghli DR, Greiser A, Frohlich M, Dietz R, Schulz-Menger J. Optimization and validation of a fully-integrated pulse sequence for modified look-locker inversion-recovery (MOLLI) T1 mapping of the heart. J Magn Reson Imaging. 2007;26:1081-6.

24. Kawel-Boehm N, Maceira A, Valsangiacomo-Buechel ER, et al. Normal values for cardiovascular magnetic resonance in adults and children. J Cardiovasc Magn Reson. 2015;17:29.

25. Lazarus A, Varin J, Ounnoughene Z, et al. Relationships among electrophysiological findings and clinical status, heart function, and extent of DNA mutation in myotonic dystrophy. Circulation. 1999;99:1041-6.

26. Colleran JA, Hawley RJ, Pinnow EE, Kokkinos PF, Fletcher RD. Value of the electrocardiogram in determining cardiac events and mortality in myotonic dystrophy. Am J Cardiol. 1997;80:1494-7.

27. Lazarus A, Varin J, Babuty D, Anselme F, Coste J, Duboc D. Long-term follow-up of arrhythmias in patients with myotonic dystrophy treated by pacing: a multicenter diagnostic pacemaker study. J Am Coll Cardiol. 2002; 40:1645-52.

28. Nguyen TP, Qu Z, Weiss JN. Cardiac fibrosis and arrhythmogenesis: the road to repair is paved with perils. J Mol Cell Cardiol. 2014;70:83-91.

29. Waller BF, Gering LE, Branyas NA, Slack JD. Anatomy, histology, and pathology of the cardiac conduction system--part III. Clin Cardiol. 1993;16:436-42.

30. Halliday BP, Baksi AJ, Gulati A, et al. Outcome in dilated cardiomyopathy related to the extent, location, and pattern of late gadolinium enhancement. JACC Cardiovasc Imaging. 2018.

31. Rau F, Freyermuth F, Fugier $C$, et al. Misregulation of miR-1 processing is associated with heart defects in myotonic dystrophy. Nat Struct Mol Biol. 2011;18:840-5.

32. Meola G, Cardani R. Myotonic dystrophies: an update on clinical aspects, genetic, pathology, and molecular pathomechanisms. Biochim Biophys Acta. 2015;1852:594-606.

33. Di Marco A, Anguera I, Schmitt M, et al. Late gadolinium enhancement and the risk for ventricular arrhythmias or sudden death in dilated cardiomyopathy: systematic review and meta-analysis. JACC Heart Fail. 2017;5:28-38.

34. Disertori M, Rigoni M, Pace N, et al. Myocardial fibrosis assessment by LGE is a powerful predictor of ventricular Tachyarrhythmias in ischemic and nonischemic LV dysfunction: a meta-analysis. JACC Cardiovasc Imaging. 2016:9:1046-55.

35. Dweck MR, Joshi S, Murigu T, et al. Midwall fibrosis is an independent predictor of mortality in patients with aortic stenosis. J Am Coll Cardiol. 2011;58:1271-9. 
36. Gulati A, Jabbour A, Ismail TF, et al. Association of fibrosis with mortality and sudden cardiac death in patients with nonischemic dilated cardiomyopathy. JAMA. 2013;309:896-908.

37. Al-Khatib SM, Stevenson WG, Ackerman MJ, et al. 2017 AHA/ACC/HRS guideline for Management of Patients with Ventricular Arrhythmias and the prevention of sudden cardiac death: executive summary: a report of the American College of Cardiology/American Heart Association task force on clinical practice guidelines and the Heart Rhythm Society. J Am Coll Cardiol. 2018;72:1677-749.

38. Raman SV, Hor KN, Mazur W, et al. Eplerenone for early cardiomyopathy in Duchenne muscular dystrophy: a randomised, double-blind, placebocontrolled trial. Lancet Neurol. 2015;14:153-61.

39. Sansone VA, Brigonzi E, Schoser B, et al. The frequency and severity of cardiac involvement in myotonic dystrophy type 2 (DM2): long-term outcomes. Int J Cardiol. 2013;168:1147-53.

40. Schmacht L, Traber J, Grieben U, et al. Cardiac involvement in myotonic dystrophy type 2 patients with preserved ejection fraction: detection by cardiovascular magnetic resonance. Circ Cardiovasc Imaging. 2016;9.

Ready to submit your research? Choose BMC and benefit from:

- fast, convenient online submission

- thorough peer review by experienced researchers in your field

- rapid publication on acceptance

- support for research data, including large and complex data types

- gold Open Access which fosters wider collaboration and increased citations

- maximum visibility for your research: over $100 \mathrm{M}$ website views per year

At $\mathrm{BMC}$, research is always in progress.

Learn more biomedcentral.com/submissions 